\title{
D.H. Lehmer's Tridiagonal determinant: An Étude in (Andrews-Inspired) Experimental Mathematics
}

Shalosh B. EKHAD and Doron ZEILBERGER

Dedicated to George Andrews on his 80th birthday

\section{Lehmer's Theorem and its Finite Form}

Define, with Lehmer ([L], p. 54), $M(n)=M(n)(X, q)$, to be the following tridiagonal $n \times n$ matrix (we changed $a$ to $\sqrt{X}$ and $r$ to $q$ )

$$
M(n)_{i, j}=\left\{\begin{array}{l}
1 \quad \text { if } i-j=0 \\
\sqrt{X} q^{(i-1) / 2} \text { if } i-j=-1 \\
\sqrt{X} q^{(i-2) / 2} \text { if } i-j=1 \\
0 \text { otherwise. }
\end{array}\right.
$$

Theorem 1 (Lehmer [L])

$$
\lim _{n \rightarrow \infty} \operatorname{det} M(n)(X, q)=\sum_{a=0}^{\infty} \frac{(-1)^{a} X^{a} q^{a(a-1)}}{(1-q)\left(1-q^{2}\right) \cdots\left(1-q^{a}\right)}
$$

(As noted by Lehmer, when $X=-q$ and $X=-1$ one gets the sum sides of the famous Rogers-Ramanujan identities.)

Our new result is an explicit expression for the finite form, that immediately implies Lehmer's theorem, by taking the limit $n \rightarrow \infty$, and gives it a new (and shorter!) proof.

\section{Theorem 2}

$$
\operatorname{det} M(n)(X, q)=\sum_{a=0}^{\lfloor n / 2\rfloor} \frac{(-1)^{a} X^{a} q^{a(a-1)}\left(1-q^{n-a}\right)\left(1-q^{n-a-1}\right) \cdots\left(1-q^{n-2 a+1}\right)}{(1-q)\left(1-q^{2}\right) \cdots\left(1-q^{a}\right)}
$$

Proof of Theorem 2: As noted by Lehmer ([L], Eq. (3)), by expanding with respect to the last row, we have

$$
\operatorname{det} M(n)(X, q)=\operatorname{det} M(n-1)(X, q)-X q^{n-2} \operatorname{det} M(n-2)(X, q) \cdot(\text { LehmerRecurrence) }
$$

Using the $q$-Zeilberger algorithm ${ }^{1}$ ([PWZ],[Z], see also $[\mathrm{PR}]$ for a nice Mathematica version), we see that the right side of Theorem 2 also satisfies the very same recurrence. Since it holds for the initial conditions $n=1$ and $n=2$ (check!), the theorem follows by induction.

1 Typing qzeil((-1)**a* $\left.\mathrm{X}^{* *} \mathrm{a}^{*} \mathrm{q}^{* *}\left(\mathrm{a}^{*}(\mathrm{a}-1)\right)^{*} \mathrm{qbin}(\mathrm{n}-\mathrm{a}, \mathrm{a}), \mathrm{S}, \mathrm{a}, \mathrm{n},[]\right)$; in $\mathrm{qEKHAD}$ gives $X q^{n}-S+S^{2}, X q^{n}$ that is the recurrence operator annihilating the sum ( $S$ is the forward shift operator in $n$ ) followed by the 'certificate' (i.e. the proof, see $[\mathrm{PWZ}]))$. 


\section{Secrets form the Kitchen}

Our paper could have ended here. We have increased human knowledge by extending a result of a famous number theorist, and proved it rigorously. But, at least as interesting as the statement of the theorem (and far more interesting than the proof) is the way it was discovered, and the rest of this paper will consist in describing two ways of doing it. The first way is a direct adaptation of George Andrews' "reverse-engineering" approach beautifully illustrated in the last chapter of his delightful booklet [A] (based on 10 amazing lectures, given at Arizona State University, May 1985 that we were fortunate to attend). In that masterpiece (section 10.2) he described how he used the computer algebra system SCRATCHPAD to prove a deep conjecture by three notable mathematicians: George Lusztig, Ian Macdonald and C.T.C. Wall. In Andrews' approach, it is assumed that the discoverer knows about Gaussian polynomials, and knows how to spot them. In other words the 'atoms' are Gaussian polynomials. In the second, more basic, approach, the only pre-requisite is the notion of polynomials, and Gaussian polynomials pop-up naturally in the act of discovery.

We will start completely from scratch, pretending that we did not read Lehmer's paper. In fact we did not have to 'pretend'. We had no clue that Lehmer's paper existed until way after we discovered (and proved) Theorem 2, (and hence reproved Lehmer's Theorem 1). This is the time for a short "commercial break", since this paper (like so many other ones!) owes it existence to the OEIS.

[Start of commercial break.]

\section{Serendipity and the OEIS}

We learned about Lehmer's Theorem 1 via serendipity, thanks to that amazing tool that we are so lucky to have, the On-Line Encyclopedia of Integer Sequences [S] (OEIS).

Recall that a composition of $n$ is an array of positive integers $\left(p_{1}, \ldots, p_{k}\right)$ such that $p_{1}+\ldots+p_{k}=n$, and they are very easy to count (there are $2^{n-1}$ of them). A partition of $n$ is a composition with the additional property that it is weakly decreasing, i.e.

$$
p_{i}-p_{i+1} \geq 0 \quad(1 \leq i<k \quad),
$$

(and they are much harder to count) .

My current PhD student, Mingjia Yang $[\mathrm{Y}]$ is investigating relaxed partitions, that she calls $r$ partitions, that are compositions of $n$ with the condition

$$
p_{i}-p_{i+1} \geq r
$$

When $r=1$ we get the familiar partitions into distinct parts, and when $r=2$ we get one of the actors in the Rogers Ramanujan identities. But what about negative $r$ ? In particular what about $(-1)$-partitions? After generating the first twenty terms

$$
1,2,4,7,13,23,41,72,127,222,388,677,1179,2052,3569,6203,10778,18722,32513,56455 \quad \text {, }
$$


we copied-and-pasted it to the OEIS, and sure enough, we were scooped! It is sequence A003116, whose (former) description was 'reciprocal of an expansion of a determinant', that pointed to sequence A039924, mentioning Lehmer's Theorem 1 (in fact the special case $X=q$ ). As a reference it cited 'personal communication' by Herman P. Robinson, a friend and disciple of Lehmer. The OEIS entry for A039924 also referenced Lehmer's "lecture notes on number theory" but we could not find it either on-line or off-line.

Since Lehmer's proof seemed to have been lost, we tried to prove it ourselves, and succeeded. Our approach, inspired by Andrews' [A], was to first find an explicit expression for the finite form, and then take the limit as $n$ goes to infinity (like Andrews did for the L-M-W conjecture). Only after we had the proof, we searched MathSciNet for

"Lehmer AND determinant AND tridiagonal" ,

and discovered [L]. To our relief, Lehmer's proof was longer than ours, and did not go via the finite form, Theorem 2. As far as we know, Theorem 2 is new. Once we discovered the reference [L] we notified Neil Sloane, and he added that reference to the relevant sequences $\mathbf{A 0 0 3 1 1 6}$ and A039924. So the present paper is yet another paper that owes its existence to the OEIS!

[End of commercial break.]

\section{How the Statement of Theorem 2 would have been (easily!) discovered by George Andrews}

In Andrews' proof of the L-M-W conjecture, he used the Gaussian polynomials (aka as q-binomial coefficients) as building blocks. With his approach, Theorem 2 could have been found by him fairly quickly. Let $Q_{n}(X, q):=\operatorname{det} M(n)(X, q)$.

Recall that the Gaussian polynomials $G P(m, n)(q)$ are defined by

$$
G P(m, n)(q):=\frac{\left(1-q^{m+1}\right)\left(1-q^{m+2}\right) \cdots\left(1-q^{m+n}\right)}{(1-q) \cdots\left(1-q^{n}\right)}
$$

(in spite of their look, they are polynomials!) .

The way George Andrews would have discovered Theorem 2 is as follows.

Initially, crank out the first, say, twenty terms of the sequence of polynomials $Q_{n}(X, q)$, either by evaluating the determinants, or, more efficiently, via (LehmerRecurrence).

You don't need a computer to realize that the coefficient of $X^{0}$, i.e. the constant term, is always 1.

The coefficients of $X=X^{1}$ in $Q_{n}(X, q)$ for $n$ from 1 to 8 are

$\left[0,-1,-1-q,-1-q-q^{2},-1-q-q^{2}-q^{3},-1-q-q^{2}-q^{3}-q^{4},-1-q-q^{2}-q^{3}-q^{4}-q^{5},-1-q-q^{2}-q^{3}-q^{4}-q^{5}-q^{6}\right]$.

A quick glance by George Andrews would have made him conjecture that it is

$$
-G P(n-2,1)(q) \text {. }
$$


Moving right along, here are the coefficients of $X^{2}$ for $1 \leq n \leq 10$ :

$$
\begin{gathered}
{\left[0,0,0, q^{2}, q^{2}+q^{3}+q^{4}, q^{2}+q^{3}+2 q^{4}+q^{5}+q^{6}, q^{2}+q^{3}+2 q^{4}+2 q^{5}+2 q^{6}+q^{7}+q^{8},\right.} \\
q^{2}+q^{3}+2 q^{4}+2 q^{5}+3 q^{6}+2 q^{7}+2 q^{8}+q^{10}+q^{9}, q^{2}+q^{3}+2 q^{4}+2 q^{5}+3 q^{6}+3 q^{7}+3 q^{8}+q^{11}+2 q^{10}+2 q^{9}+q^{12}, \\
\left.q^{2}+q^{3}+2 q^{4}+2 q^{5}+3 q^{6}+3 q^{7}+4 q^{8}+2 q^{11}+3 q^{10}+3 q^{9}+2 q^{12}+q^{13}+q^{14}\right] .
\end{gathered}
$$

Dividing by $q^{2}$ and checking against the Gaussian polynomials 'data base', suggests that the coefficient of $X^{2}$ is always

$$
q^{2} G P(n-4,2)(q)
$$

Similarly, the coefficient of $X^{3}$ would have emerged as

$$
-q^{6} G P(n-6,3)(q)
$$

The coefficient of $X^{4}$ would have emerged as

$$
q^{12} G P(n-8,4)(q)
$$

The coefficient of $X^{5}$ would have emerged as

$$
-q^{20} G P(n-10,5)(q)
$$

And bingo, it requires no great leap of an Andrews' imagination to conjecture that

$$
Q_{n}(X, q)=\sum_{a=0}^{\lfloor n / 2\rfloor}(-1)^{a} X^{a} q^{a(a-1)} G P(n-2 a, a)(q)
$$

that is identical to the statement of Theorem 2 .

\section{How the Statement of Theorem 2 could have been discovered by someone who is NOT George Andrews?}

Suppose that you have never heard of the Gaussian polynomials. You still could have conjectured the statement of Theorem 2. Even if you have never heard of Gaussian polynomials, you probably did hear of polynomials. So assuming the ansatz that, for each $a$, the coefficient of $X^{a}$ is a certain polynomial in $q^{n}$, try and fit it with a 'generic' polynomial with undetermined coefficients. ${ }^{2}$

Setting $N=q^{n}$, your computer would have guessed the following polynomial expressions (in $N=$ $\left.q^{n}\right)$ for the first five coefficients of $X$ in $Q_{n}(X)$.

2 You start out with a generic polynomial of degree 0, and keep raising the degree until success (or failure). 
- The coefficient of $X$ in $Q_{n}(X, q)$ is

$$
\frac{N-q}{q(1-q)}
$$

- The coefficient of $X^{2}$ in $Q_{n}(X, q)$ is

$$
\frac{\left(N-q^{2}\right)\left(N-q^{3}\right)}{q^{3}(1+q)(1-q)^{2}}
$$

- The coefficient of $X^{3}$ in $Q_{n}(X, q)$ is

$$
-\frac{\left(N-q^{3}\right)\left(N-q^{4}\right)\left(N-q^{5}\right)}{q^{6}(1+q)\left(q^{2}+q+1\right)(q-1)^{3}} .
$$

- The coefficient of $X^{4}$ in $Q_{n}(X, q)$ is

$$
\frac{\left(N-q^{4}\right)\left(N-q^{5}\right)\left(N-q^{6}\right)\left(N-q^{7}\right)}{q^{10}\left(q^{2}+1\right)(q-1)^{4}(1+q)^{2}\left(q^{2}+q+1\right)} .
$$

- The coefficient of $X^{5}$ in $Q_{n}(X, q)$ is

$$
-\frac{\left(N-q^{5}\right)\left(N-q^{6}\right)\left(N-q^{7}\right)\left(N-q^{8}\right)\left(N-q^{9}\right)}{q^{15}(q-1)^{5}\left(q^{4}+q^{3}+q^{2}+q+1\right)(1+q)^{2}\left(q^{2}+q+1\right)\left(q^{2}+1\right)} .
$$

This immediately leads one to guess that the numerator is always

$$
(-1)^{a}\left(N-q^{a}\right)\left(N-q^{a+1}\right) \cdots\left(N-q^{2 a-1}\right) .
$$

On the other hand, the sequence of denominators, let's them call them $d(a)$, for $1 \leq a \leq 5$, happens to be

$$
\begin{gathered}
{\left[-q(q-1), q^{3}(1+q)(q-1)^{2},-q^{6}(1+q)\left(q^{2}+q+1\right)(q-1)^{3}, q^{10}\left(q^{2}+1\right)(q-1)^{4}(1+q)^{2}\left(q^{2}+q+1\right)\right.} \\
\left.,-q^{15}(q-1)^{5}\left(q^{4}+q^{3}+q^{2}+q+1\right)(1+q)^{2}\left(q^{2}+q+1\right)\left(q^{2}+1\right)\right] .
\end{gathered}
$$

This looks a bit complicated, but let's form the sequence of ratios $d(a) / d(a-1)$ for $a=2,3,4,5$ and expand, getting

$$
\left[q^{2}-q^{4}, q^{3}-q^{6},-q^{8}+q^{4}, q^{5}-q^{10}\right],
$$

that is clearly $q^{a}\left(1-q^{a}\right)$. Hence the coefficient of $X^{a}$ in $Q_{n}(X, q)$ is guessed to be

$$
\frac{(-1)^{a}\left(N-q^{a}\right)\left(N-q^{a+1}\right) \cdots\left(N-q^{2 a-1}\right)}{q^{a(a+1) / 2}(1-q) \cdots\left(1-q^{a}\right)} .
$$

By putting $N=q^{n}$ we get the statement of Theorem 2 . 
So with this second approach, we discovered the Gaussian polynomials ab initio, our only gamble was that the coefficients of $X$ in $Q_{n}(X, q)$ are always polynomials in $q^{n}$.

\section{Concluding words}

Let us quote the last sentence of section 10.2 of $[\mathrm{A}]$, where Andrews described his pioneering (experimental mathematics!) approach illustrated by his discovery process of the proof of the L-M$\mathrm{W}$ conjecture.

"From here the battle with the $L-M-W$ conjecture is 90 percent won. Standard techniques allow one to establish the [finite form] of the conjecture, and a simple argument leads to the original conjecture."

Today the 90 percent may be replaced by 99.999 percent, since the final verification can be done automatically by using the so-called $q$-Zeilberger algorithm ([PWZ] $[\mathrm{Z}][\mathrm{PR}])$. In the much more difficult L-M-N case, this would have saved George Andrews a few hours, and would have made it accessible to anyone else. In the present case, you can still use the $q$-Zeilberger algorithm, if you are feeling lazy, but it is not too hard to do it purely humanly. Can you do it?

\section{References}

[A] George E. Andrews, " $q$-Series: Their Development and Applications in Analysis, Number Theory, Combinatorics, Physics, and Computer Algebra", CBMS \#66, American Mathematical Society, 1986.

[L] Derrick H. Lehmer, Combinatorial and cyclotomic properties of certain tridiagonal matrices, Proceedings of the Fifth Southeastern Conference on Combinatorics, Graph Theory, and Computing (Florida Atlantic University, Boca Raton, Fla. 1974)), 53-74. Congressus Numerantium, No. X, Utilitas Math., Winnipeg., Man. 1974. MR0441852(56 \#243) . A scanned copy is available from

http://sites.math.rutgers.edu/ zeilberg/akherim/LehmerDet1974.pdf [Accessed Aug. $18,2018]$.

[PR] Peter Paule and Axel Riese, A Mathematica q-analogue of Zeilberger's algorithm based on an algebraically motivated approach to q-hypergeometric Telescoping, in: "Special Functions, q-Series and Related Topics," Fields Inst. Commun. 14, 179-210, 1997 .

Available from: http://www.risc.jku.at/publications/download/risc_118/diss.pdf [Accessed Aug. 18, 2018].

[PWZ] Marko Petkovšek, Herbert S. Wilf, and Doron Zeilberger, " $A=B$ ", A.K. Peters, 1996. Available from: https://www.math.upenn.edu/ ${ }^{w i l f / D o w n l d . h t m l ~[A c c e s s e d ~ A u g . ~ 18, ~ 2018] . ~}$

[S] Neil A. J. Sloane, The On-line Encyclopedia of Integer Sequences , https://oeis.org . [Y] Mingjia Yang, Relaxed partitions, in preparation. 
[Z] Doron Zeilberger, qEKHAD (a Maple program implementing the $q$-Zeilberger algorithm). Available from

http://sites.math.rutgers.edu/〜zeilberg/tokhniot/qEKHAD [Accessed Aug. 18, 2018].

Shalosh B. Ekhad, c/o D. Zeilberger, Department of Mathematics, Rutgers University (New Brunswick), Hill Center-Busch Campus, 110 Frelinghuysen Rd., Piscataway, NJ 08854-8019, USA.

Email: ShaloshBEkhad at gmail dot com .

Doron Zeilberger, Department of Mathematics, Rutgers University (New Brunswick), Hill CenterBusch Campus, 110 Frelinghuysen Rd., Piscataway, NJ 08854-8019, USA.

Email: DoronZeil at gmail dot com . 